\title{
The role of airflow for the relief of chronic refractory breathlessness
}

\section{Flavia Swan ${ }^{1}$ and Sara Booth ${ }^{2}$}

${ }^{1}$ SEDA Research group, Hull York Medical School, University of Hull, Hull HU6 7RX.

${ }^{2}$ Palliative Care Team, Cambridge University Hospitals' NHS Foundation Trust, Addenbrooke's Hospital, Hills Rd, Cambridge CB2 0QQ.

\section{Correspondence to}

Flavia Swan, SEDA Research group, Hull York Medical School, University of Hull, Hull HU6 7RX.

E-mail hyfes@hyms.ac.uk

\section{Purpose of review}

Chronic refractory breathlessness is a major cause of suffering to people with advanced stage and some newo legerenture

cardio-respiratory diseases. It is a frightening, distressing and disabling symptom that $\wedge$

imposes significant burdens on family members. Evidence is mounting for the role of facial or nasal airflow for the relief of chronic refractory breathlessness in those patients with mild or normoxaemia. Airflow can be delivered from the hand-held fan or from compressed medical air via a face mask or nasal cannulae. The hand-held fan offers patients a simple, low cost, self-management strategy that is not associated with any known risks. Therefore, it is timely and relevant to review the research available for the efficacy and appropriateness of facial or nasal airflow. 


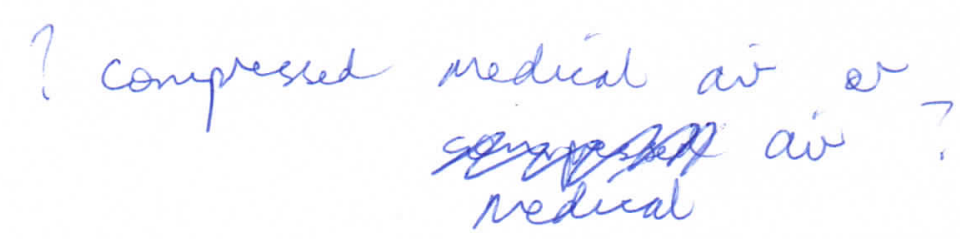

\section{Recent findings}

There is sufficient review evidence available to suggest that airflow from the hand-held fan or compressed medical air can provide clinically relevant and discernible relief of chronic refractory breathlessness at rest in patients with advanced erofoprespiratory diseases.

\section{Summary}

The hand-held fan should be considered as one of the first interventions to try in management Lyposaend

plans for patients who present with mild or normoxaemia and chronic refractory

$$
\text { a on numal exertion }
$$

breathlessness at rest. Emerging evidence indicates that airflow from the hand-held fan may a

also have an important role with exertion-induced breathlessness; decreasing distress and

speeding recovery time after exercise, thereby helping patients self-manage their symptoms

during everyday general activity and plan for crises of breathlessness, secure in the

knowledge that they have a tangible easily portable device to try in any circumstances.

\section{Keywords}

Hand-held fan, airflow, breathlessness management

\section{Introduction}

Chronic refractory breathlessness is a frequent and devastating symptom of many advanced stage afdio-Pespiratory diseases. There are now many accounts of the distressing effects of breathlessness; patients, family and close carers all commonly experience considerable physical and emotional burdens as well as social isolation $(1,2)$. Invariably the symptom worsens as the disease advances (3), and breathlessness may persist despite optimising the treatment of underlying causes; when it is defined as refractory (4). Patients and carers 
commonly describe feelings of panic with the onset of a sudden acute exacerbation of breathlessness and these often precipitate an unscheduled emergency hospital admission (5). Currently management is modelled on a multi-disciplinary and complex approach combining pharmacological and non-pharmacological interventions to target the many causes and dimensions of the symptom $(6,7)$. One non-pharmacological option identified as helping alleviate the sensation of breathlessness is the hand-held fan. The passage of cool air directed to the face, nasal mucosa or pharynx may alter ventilation and influence breathing $(8,9)$. Potential mechanisms underlying the effect of airflow are thought to relate to the stimulation of the trisgeminal skin receptors causing the diving response, or via the afferent input from nasal mucosa and upper airway "flow" receptors decreasing central respiratory drive (10-12).

\section{Patient's perception and use of airflow for relief of breathlessness}

Historically, it has long been observed that breathless patients avoid confined spaces and cite "a cold draught" or "opening a window" as successful actions to help relieye breathlessness.

A previous mixed methods study examining coping strategies in 30 COPD patients found that "getting fresh air" was ranked in the top three of the most effective and frequently used selfmanagement techniques (13). Moreover, the published studies to date that have investigated the effectiveness of the hand-held fan clearly identify sub-groups that deem the intervention highly acceptable and helpful $(14,15)$. However, patient? experience of this therapy could influence their consideration of the hand-held fan. Oxygen can be viewed as "lifeline" (16), therefore the airflow produced by a hand-held fan may not be perceived as an emergency option to relieve breathlessness. Moreover, it is possible that the simplicity of an everyday object freely available for the general public to purchase may not delineate or give the hand-held fan the physical appearance of a medical device. This to indicates the importance of the clinician's role as how the hand-held fan is introduced to a 
patient may dical

patient may directly influence their pre-conceived ideas of plausibility and it could provide the credibility necessary to try the device during drises episodes of breathlessness (17).

Clinician's ' perception and use of airflow for relief of breathlessness

Equally, the frequency of use of airflow as a treatment for breathlessness varies widely across healthcare settings. For example, airflow delivered as compressed medical air via facemask or nasal cannulae would rarely be deemed the first option to manage an acute exacerbation of chronic refractory breathlessness in the emergency department, yet in contrast specialist clinical settings such as the Breathlessness Intervention Service or the Breathlessness Support Service would consider airflow delivered from the hand-held fan a core component of any breathlessness management $(6,7)$. It is likely that the use of airflow is influenced by the clinician 35 perception of oxygen, a well-known therapy that is entrenched in our healthcare For chione Liathlessrees,

culture (18). Øne survey demonstrated that $70 \%$ of responding clinicians would prescribe oxygen irrespective of saturation levels, while a further $35 \%$ would prescribe solely on patient request (19). Moreover, the results from a large consecutive cohort study suggested that the carer may also exert an important role as patients were more likely to be prescribed oxygen if they lived with their carer than alone (20). The data indicate that hidden drivers could undermine the clinical rationale for considering oxygen and negate the possibility of offering airflow delivery from a hand-held fan or compressed cylinder medical air.

\section{Guidelines for clinical practice}

At present no clinical guidelines exist and there is no formal procedure available to identify which patients might benefit from airflow delivery. This is hardly surprising as the only Cochrane review was unable to reach any conclusions about the hand-held fan due to insufficient evidence available at the time of writing (21). 


\title{
Review of the literature
}

\author{
are Gew
}

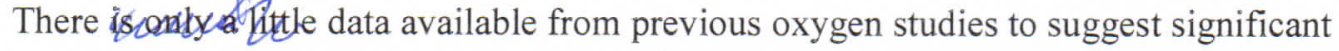
benefit of oxygen over medical comppessed air delivery for the relief of chronic refractory breathlessness in patients with mild or non-hypoxaemia (22). Systematic reviews of oxygen therapy in patients with advanced cancer or cardiac disease have consistently failed to find any additional benefit from the inhalation of oxygen versus medical air (23-25). In contrast a Cochrane review that examined the effectiveness of oxygen in COPD patients did find some benefit in comparison to the medical air delivery. A meta-analysis of 14 studies found a standardised mean difference [SMD] $-0.46(95 \%$ CI -0.59 to -0.33$), \mathrm{p}<0.00001$ when oxygen was delivered in a continuous mode or as LTOT, although the authors also advised caution in interpretation of the findings as there were many small studies, coupled with significant heterogeneity (26). Moreover, the meta-analysis did not include the now published data from the largest, international, multi-centre RCT that investigated the effectiveness of palliative oxygen in 239 participants (63\% COPD) with refractory breathlessness who received at least 15 hours a day of oxygen or medical air delivered via home concentrator for seven days during everyday general activity (27). Results found improved numerical rating scale (NRS) breathlessness intensity irrespective of the study arm, with morning and evening scores decreases of $-0.8(95 \% \mathrm{CI}-0.5$ to -1.1$)$ and $-0.4(95 \% \mathrm{CI}-0.1$ to 0.7$), \mathrm{p}<0.001$ respectively (27). This provides further evidence that the airflow in the placebo arm is an active a placebo intervention and not comparator, while the results correspond with those from another more recent study of ambulatory oxygen when used with general activity in 143 COPD patients without severe resting hypoxaemia. Again, the data reported statistically significant improvements in breathlessness in the whole study group and no differences in benefit between the groups receiving oxygen or air during the 12 week study period (28). 
Therefore, it is likely that the magnitude of oxygen benefit in COPD patients could diminish once the Cochrane review is updated to include the latest evidence from these studies.

Moreover, there are now two published RCTs that examine the effectiveness of airflow from the hand-held fans a fully powered crossover that recruited 50 in-patients with any advanced which reduction

disease diagnosis estimated a significant in $_{\Lambda} 7 \mathrm{~mm}_{\Lambda}$ Visual Analogue Scale (VAS) breathlessness ( $95 \%$ CI $2.5-11.7 \mathrm{~mm}), \mathrm{p}=0.003$ at rest from using the hand-held fan to the face for 5

minutes compared to the leg after accounting for an inadequate washout period (14), while a feasibility study reported a limited modified Borg breathlessness score change of - 0.6 (SD 2.1) after 2 months use of the hand-held fan with general activity in 70 out-patients $(65 \%$ COPD, 35\% cancer ) (15). The Minimal Clinically Important Difference (MCID) for patients with chronic breathlessness from a variety of conditions is known as -1 point Borg score or 9mm VAS change (29-31). Therefore, the benefit derived from the hand-held fan at rest suggests a level of improvement that is discernible to the patient and would influence choice of intervention, while the results from the feasibility study do not substantiate the same effectiveness of using the hand-held fan with everyday general activity. However, a previous study did find a transient but significant reduction in breathlessness from a fan blowing onto the face in addition to the flow of oxygen from a nasal cannula on day one during 3 subsequent days of exercise tests in 17 COPD patients (32).

Furthermore, two other prior studies have demonstrated the possibility of increased exercise tolerance with the use of cold air in patients with respiratory disease. Marchetti et al reported improved performance of a leg ergometer test with a large fan directed to the face in comparison to the leg, but no difference in the breathlessness intensity experienced in 4 COPD patients using a randomised cross-over design (33). A further RCT of 19 COPD 


\section{How evidence is developing in last 2 years?}

Recently the results from a hand-held fan cohort study $(n=31)$ found a mean $-12.8 \mathrm{~mm}$, (SD $\pm 20.7)$ VAS breathlessness following 5 minutes use of the hand-held fan to the face at rest (35); results that correlate with the previous crossover RCT findings and provide further evidence of the significant benefit of airflow when used at rest in patients with mild or normoxaemia and chronic refractory breathlessness. In addition, a preliminary exploration into the feasibility of using magnetoencephalography (MEG) scanning for patients with chronic refractory breathlessness found that post-exercise during recovery the pattern of alpha activity in the parietal-temporal regions seemed to change and decrease when airflow was used (36). Mis paper is now accepted or pubbiabion However, gaps in the evidence and questions remain about the role of airflow with general activity or exercise and the potential use in an emergency crisis plan. The previous study of general activity was unable to demonstrate significant benefit of the hand-held fan although limited by power as a Phase II design (15), it is possible that the measurement of Borg breathlessness intensity over the last 24 hours did not appropriately reflect any patient improvement in exercise tolerance. An issue highlighted by a previous paper as breathlessness scores may remain static or worsen after the initial introduction of an intervention to alleviate symptoms because patients are able to exert themselves to the same level of breathlessness without knowing that their exercise tolerance has changed (37). The challenge to identify the appropriate outcome measurements to reflect breathlessness improvements related to changes in exercise tolerance are now being addressed by two 
feasibility studies; Fan, Activity and Breathlessness (FAB), Australian Clinical Trials Register ACTRN1261400052568, and Calming Hand and Fan Feasibility (CHAFF), Clinical Trial Register ISRCTN40230190. Both have recently completed recruitment and explore the level of variability around a range of outcome measurements when the hand-held fan is used with everyday general activity and exercise.

\section{Implications for practice and research}

It is clear that the clinical role of airflow and the use of the hand-held fan with activity and exercise is still unfolding. It is known that the trajectory of advanced cardio-respiratory diseases may lengthen and people are now living longer with chronic illness (3). Equally, there is strong evidence that the peripheral skeletal muscle changes occurring in COPD, cancer and other chronic diagnoses are important in the perpetuation of breathlessness (3841). Therefore, the level of physical activity and function in patients with advanced cardiorespiratory diseases is one of the primary concerns in the management of chronic refractory breathlessness. Problems may arise if exertion induced breathlessness discourages patients from persisting with normal everyday activity or exercise because of its unpleasantness or the belief that it may be harmful (42). This is highlighted by pulmonary rehabilitation as strong evidence of benefit exists in the management of COPD patients (43), with improvements documented in exercise tolerance, exertion induced breathlessness and a reduction in the

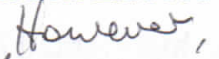

number of hospitalisations (44-47). Q $u 4 t$, most programmes are associated with high drop-out rates, patient adherence problems and benefits that diminish over time (48-52).

Therefore, the airflow from a hand-held fan could have an important role in clinical practice to support patients during exercise and may decrease unpleasant sensations, improve selfefficacy or speed recovery time from exertion induced breathlessness. It could also be used to 
intervene with symptom control at an earlier stage during chronic illness and promote the benefits of continued activity over time. Incremental benefit may occur with the cumulative use of different interventions (53), and should be considered part of a multi-dimensional strategy for breathlessness management. It is possible that the addition of an intervention such as the hand-held fan could make the difference between a patient being able to go up and down stairs and reach the bathroom safely, thereby continuing to manage activities of daily living in their own environment. It is rare to have easy access to a cheap, portable, intervention that is potentially useful globally, and that enhances self -efficacy and reduces the impact of a distressing symptom without known adverse effects. It may also help to reduce the distress of carers by giving them a specific role in an acute exacerbation of breathlessness. More work is needed urgently to confirm these emerging findings and, when confirmed, to disseminate its usefulness more widely.

\section{Conclusions}

The role of airflow is complicated by the interplay and complexity of our beliefs and culture that surround the use of oxygen therapy. Indiscriminate use of oxygen in "out of hours"

$$
\text { , in the emergercy room or by anbulare soff }
$$

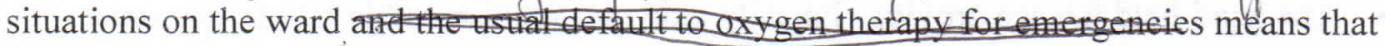
i

airflow could easily be overlooked as an option to treat breathlessness in some healthcare settings. The simplicity of the hand-held fan could influence both the patient's and clinician's perception of the intervention therefore how it is delivered may be a key issue in the acceptability of this device in the management of breathlessness (17). In clinical practice the hand-held fan offers a portable, inexpensive patient option that could increase self-efficacy, improve exercise tolerance and assist recovery from exertion induced breathlessness. It should be considered as one of the first interventions to try in the context of a management plan for patients with advanced chronic disease who present with mild or normoxaemia and 
chronic refractory breathlessness problems at rest or that interfere with everyday activities. Moreover, a portable device that provides an instantaneous effect should be a pre-requisite for all emergency situations, providing the patient with the physicality of an object and a ritual of "something to do" in the event of a crisis episode of breathlessness.

\section{Acknowledgements}

None

\section{Financial support and sponsorship}

None

\section{Conflicts of interest}

None

\section{Key Points}

- The hand-held fan can provide clinically relevant and discernible relief of chronic refractory breathlessness at rest in patients with mild or normoxaemia.

- The acceptability of the hand-held fan by clinicians is complicated by longstanding complex beliefs and culture that surround the use of oxygen therapy.

- The acceptability and effectiveness of the hand-held fan for patients may depend on how the intervention is delivered.

- The hand-held fan is a cheap, portable easily obtainable device that could help patients manage exertion induced breathlessness during daily activities. 